\title{
Exact and Approximate Symbol Error Probability of cooperative systems with best relay selection and all participating relaying using Amplify and Forward or Decode and Forward Relaying over Nakagami-m fading channels
}

\author{
Nadhir Ben Halima ${ }^{1 *}$, Hatem Boujemaa ${ }^{2}$ \\ ${ }^{1}$ College of Computer Science and Engineering in Yanbu \\ Taibah University, Madinah , Saudi Arabia \\ ${ }^{2}$ Higher School of Communications of Tunis, COSIM Research Laboratory \\ Route de Raoued Km 3.5, 2083 El Ghazala, Ariana, Tunisia \\ nbenhalima@taibahu.edu.sa, boujemaa.hatem@supcom.tn \\ *Corresponding author: Nadhir Ben Halima
}

Received December 10, 2016; revised March 1, 2017; revised April 11, 2017; accepted September 28, 2017; published January 31, 2018

\begin{abstract}
In this paper, we derive the theoretical Symbol Error Probability (SEP) of cooperative systems with best relay selection for Nakagami-m fading channels. For Amplify and Forward (AF) relaying, the selected relay offers the best instantaneous Signal to Noise Ratio (SNR) of the relaying link (source-relay-destination). In cooperative networks using Decode and Forward (DF), the selected relay offers the best instantaneous SNR of the link between the relay and the destination among the relays that have correctly decoded the transmitted information by the source. In the second part of the paper, we derive the SEP when all participating AF and DF relaying is performed. In the last part of the paper, we extend our results to cognitive radio networks where there is interference constraints : only relays that generate interference to primary receiver lower than a predefined threshold $\mathrm{T}$ can transmit. Both AF and DF relaying with and without relay selection are considered.
\end{abstract}

Keywords: Cooperative Diversity, Amplify and Forward, Decode and Forward, Nakagami-m channel, Cognitive Radio networks. 


\section{Introduction}

Cooperative diversity is a new promising concept [1-4] that allows to create a virtual MIMO (Multiple Input Multiple Output) system without requiring multiple antennas at the transmitter. It consists in using the antennas of neighboring relays to forward the transmitted signal by the source. Cooperative protocols are usually classified into two categories : the Amplify and Forward (AF) scheme in which the relay amplifies its received signal and the Decode and Forward (DF) scheme where the relay decodes and re-encodes the received signal. Bit Error Probability of cooperative systems using AF or DF strategy have been evaluated in [5]-[6].

\section{Related Work}

These conventional cooperative protocols require $\mathrm{N}+1$ channel resources, i.e. one for the source and $\mathrm{N}$ for the relays, which reduce the system spectral efficiency. To solve this problem, a new cooperation protocol called Selection AF (S-AF) was introduced in [7]. In S-AF, only the relay with the highest SNR of the relaying link (Source-Relay-Destination) is chosen as the active relay. It has been shown in [7] that S-AF offers better performance than conventional AF. An asymptotic BEP of S-AF for Rayleigh fading channels has been derived in [7]. A more accurate lower bound of the BEP of S-AF for Rayleigh fading channels has been derived in [8]. However, the exact SEP of S-AF has not been yet derived and there is no study for Nakagami-m fading channels.

In [9], the capacity outage probability of S-DF has been derived. In S-DF, the selected relay has the highest SNR of the relay to destination link among the relays that have correctly decoded. To the best of our knowledge, the BEP of S-DF has not been yet derived for Rayleigh or Nakagami-m fading channels.

More recent papers studied the performance of cognitive radio networks in the presence of Nakagami fading channels [23-30]. Cognitive radio networks improves the spectrum utilization. Primary users (licensed users) and secondary users (unlicensed) share the same spectrum. In underlay cognitive radio networks, the generated interference from secondary users to primary users should be below a predefined threshold in order to not degrade the QoS (Quality of Service) at the primary receiver. In [23-24, 26-28], AF relaying with a single AF relay was considered. Multihop relaying was studied in [25] in the presence of interference constraints. Secrecy performance of cognitive radio networks with DF relaying was investigated in [30]. A network containing a single DF relay was studied in [30].

The innovation of the paper are as follows :

- Derive the exact SEP of cooperative systems using Selective AF or Selective DF relaying for Nakagami-m fading channels. The different links are assumed to be i.ni.d. Exact SEP is not available in the literature.

- Study both all participating relaying and relay selection and derive the diversity order.

- Derive lower and upper bound of the SEP when AF relaying is used.

- Study cognitive radio networks with multiple relays. Previous studies consider a single relay [23-24, 26-30].

- Derive the diversity multiplexing tradeoff for underlay cognitive radio networks. To the best of our knowledge it is not available in the literature. 
Sections 3 and 4 study selective AF and DF relaying for Nakagami channels. Sections 5 and 6 consider all participating AF and DF relaying. Cognitive radio networks are analyzed in sections 7 and 8 . Simulation and theoretical results are given in section 9. Conclusions and perspectives are given in section 10.

\section{BEP of cooperative S-AF}

As shown in Fig. 1, we consider a wireless communication system with $\mathrm{N}$ relays Ri, a source $\mathrm{S}$ and a destination $\mathrm{D}$. In the first phase of the transmission, $\mathrm{S}$ broadcasts the signal $\mathrm{x}$ to $\mathrm{D}$ and all the relays Ri. In the second phase, the relay which offers the highest total SNR of the relaying link S-R-D is selected. The selected relay Rsel amplifies the received signal and forwards it to D. Relay selection requires some signalization and is performed by a central node. Some signalization is sent from the central node to activate the best relay. Both the Channel State Information (CSI) of first and second hop of different relays are sent to the central node (2N CSI are required were $\mathrm{N}$ is the number of relays). The central node uses the CSI of first and second hop to deduce the end-to-end SNR for each relay using (3). Then, the central node compares these end-to-end SNR and sends some signalization to activate the best one with the largest end-to-end SNR. Signalization is the drawaback of relay selection.

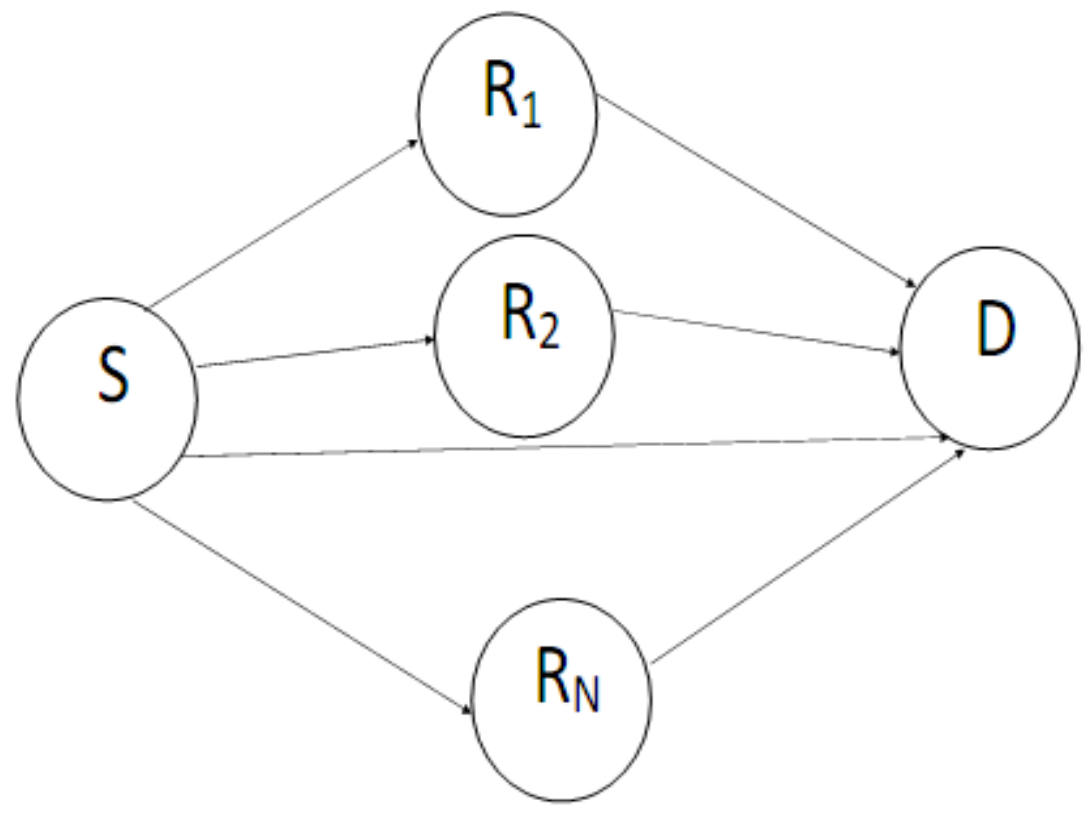

Fig. 1. System model.

Assuming that $\mathrm{D}$ combines the signals received from the source and the selected relay using a Maximum Ratio Combining (MRC) approach, the SNR at D is given by [5,7]

$$
\Gamma_{D}=\Gamma_{S, D}+\Gamma_{S, R_{s e l}, D},
$$


where

$$
\begin{aligned}
& \Gamma_{S, R_{s e l}, D}=\max _{1 \leq i \leq N} \Gamma_{S, R_{i}, D} \\
& \Gamma_{S, R_{i}, D}=\frac{\Gamma_{S, R_{i}} \Gamma_{R_{i}, D}}{\Gamma_{S, R_{i}}+\Gamma_{R_{i}, D}+1}, \\
& \Gamma_{S, D}=\frac{E_{0}}{N_{S, D}}\left|f_{S, D}\right|^{2}, \Gamma_{S, R_{i}}=\frac{E_{0}}{N_{S, R_{i}}}\left|f_{S, R_{i}}\right|^{2}, \Gamma_{R_{i}, D}=\frac{E_{i}}{N_{R_{i}, D}}\left|f_{R_{i}, D}\right|^{2}
\end{aligned}
$$

$\Gamma_{\mathrm{XY}}$ is the SNR between nodes $\mathrm{X}$ and $\mathrm{Y}, \mathrm{f}_{\mathrm{XY}}$ is the channel coefficient, $\mathrm{E}_{0}$ (resp. $\mathrm{E}_{\mathrm{i}}$ ) is the transmitted energy per symbol by source (resp. relay $\mathrm{Ri}$ ), $\mathrm{N}_{\mathrm{X}, \mathrm{Y}}$ is the noise power spectral density of link X-Y. The BEP is therefore given by

$$
P_{e}^{S-A F}=A \iint Q(\sqrt{B(x+u)}) p_{\Gamma_{S, D}}(x) p_{\Gamma_{S, R_{s e l}, D}}(u) d x d u
$$

where $A$ and $B$ depend on the considered modulation (for example, $A=1, B=2$ for $B P S K), Q(x)$ is the Marcum Q-function, $\mathrm{p}_{\mathrm{x}}(\mathrm{x})$ is the Probability Density Function (PDF) of X.

For Nakagami-m fading channels, we have

$$
p_{\Gamma_{S, D}}(x)=\left(\frac{m_{S, D}}{\bar{\Gamma}_{S, D}}\right)^{m_{S, D}} \frac{x^{m_{S, D}-1}}{\Gamma\left(m_{S, D}\right)} e^{-\frac{m_{S, D}}{\bar{\Gamma}_{S, D}}}, x \geq 0
$$

$\Gamma($.$) is the gamma function, \mathrm{m}_{\mathrm{S}, \mathrm{D}}$ is the fading figure and

$$
\bar{\Gamma}_{S, D}=E\left(\Gamma_{S, D}\right)
$$

$\mathrm{E}(\mathrm{Y})$ is the expectation of $\mathrm{Y}$.

$$
p_{\Gamma_{S, R_{s e l}, D}}(x)=\sum_{i=1}^{N} p_{\Gamma_{S, R_{i}, D}}(x) \prod_{j=1, j \neq i}^{N} P_{\Gamma_{S, R_{j}, D}}(x),
$$

$\mathrm{P}_{\mathrm{X}}(\mathrm{x})$ is the Cumulative Distribution Function (CDF) of X. The CDF has been derived in [10] (equation (12)). By taking the derivative of the CDF, we obtain 


$$
\begin{aligned}
& p_{\Gamma_{S, R_{i}, D}}(x)=\frac{-2\left(m_{R_{i}, D}\right)^{m_{R_{i}, D}}\left(m_{S, R_{i}}-1\right) !}{\left(\bar{\Gamma}_{R_{i}, D}\right)^{m_{R_{i}, D}} \Gamma\left(m_{S, R_{i}}\right) \Gamma\left(m_{R_{i}, D}\right)} \exp \left[-x\left(\frac{m_{S, R_{i}}}{\bar{\Gamma}_{S, R_{i}}}+\frac{m_{R_{i}, D}}{\bar{\Gamma}_{R_{i}, D}}\right)\right] \\
& \times\left[-\left(\frac{m_{S, R_{i}}}{\bar{\Gamma}_{S, R_{i}}}+\frac{m_{R_{i}, D}}{\bar{\Gamma}_{R_{i}, D}}\right) D(x)+\sum_{k=0}^{m_{S, R_{i}}-1} \sum_{l=0}^{k} \sum_{r=0}^{m_{R_{i}, D}-1}\left\{\frac{C_{k}^{l} C_{m_{R_{i}, D}-1}^{r}}{k !}\right.\right. \\
& \times\left(\frac{m_{R_{i}, D}}{\bar{\Gamma}_{R_{i}, D}}\right)^{(l-r-1) / 2}\left(\frac{m_{S, R_{i}}}{\bar{\Gamma}_{S, R_{i}}}\right)^{(2 k-l+r-1) / 2} x^{\left(2 k+2 m_{R_{i}, D}-l-r-3\right) / 2}(x+1)^{(l+r-1) / 2} \\
& \times\left[K_{l-r-1}\left(2 \sqrt{\frac{m_{R_{i}, D} m_{S, R_{i}}(1+x) x}{\bar{\Gamma}_{R_{i}, D} \bar{\Gamma}_{S, R_{i}}}}\right)\left((r+1) x+\left(k+m_{R_{i}, D}-l\right)(x+1)\right)\right. \\
& -\sqrt{x(x+1)}(1+2 x) \sqrt{\frac{m_{R_{i}, D} m_{S, R_{i}}}{\bar{\Gamma}_{R_{i}, D} \bar{\Gamma}_{S, R_{i}}}} K_{l-r-2}\left(2 \sqrt{\left.\left.\left.\frac{m_{R_{i}, D} m_{S, R_{i}}(1+x) x}{\bar{\Gamma}_{R_{i}, D} \bar{\Gamma}_{S, R_{i}}}\right)\right]\right\},},\right.
\end{aligned}
$$

where

$C_{k}^{l}=k ! /(l !(k-l) !), K_{n}($.$) is the n$-th order modified Bessel function of the second kind,

$$
\begin{gathered}
D(x)=\sum_{k=0}^{m_{S, R_{i}}-1} \sum_{l=0}^{k} \sum_{r=0}^{m_{R_{i}, D}-1}\left\{\frac{C_{k}^{l} C_{m_{R_{i}, D}-1}^{r}}{k !}\left(\frac{m_{R_{i}, D}}{\bar{\Gamma}_{R_{i}, D}}\right)^{(l-r-1) / 2}\left(\frac{m_{S, R_{i}}}{\bar{\Gamma}_{S, R_{i}}}\right)^{(2 k-l+r+1) / 2}\right. \\
\left.\quad \times x^{\left(2 k+2 m_{R_{i}, D}-l-r-1\right) / 2}(x+1)^{(l+r+1) / 2} K_{l-r-1}\left(2 \sqrt{\frac{m_{R_{i}, D} m_{S, R_{i}}(1+x) x}{\bar{\Gamma}_{R_{i}, D} \bar{\Gamma}_{S, R_{i}}}}\right)\right\} .
\end{gathered}
$$

\section{BEP of cooperative S-DF}

For S-DF relaying, each relay having correctly decoded sends to the central node some signalization so that it can be a candidate relay. The destination sends the CSI of second hop of all candidate relays to the central node. Then, the central node compares the SNR of second hop of all candidate relays and activate the best one with largest second hop SNR.

The BEP of S-DF can be written as 


$$
P_{e}^{S-D F}=\sum_{\Delta} P_{e / \Delta}^{S-D F} \operatorname{pr}(\Delta),
$$

where $\Delta$ is the set of relays that have correctly decoded,

$$
\operatorname{pr}(\Delta)=\prod_{i \in \Delta}\left(1-P e_{R_{i}}\right) \prod_{j \notin \Delta} P e_{R_{j}},
$$

$\mathrm{Pe}_{\mathrm{Ri}}$ is the average SEP at relay Ri,

$$
\begin{gathered}
P e_{R_{i}}=A\left[\frac{1-\mu_{S, R_{i}}}{2}\right]^{m_{S, R_{i}}} \sum_{k=0}^{m_{S, R_{i}}-1} C_{m_{S, R_{i}}+k-1}^{k}\left(\frac{1+\mu_{S, R_{i}}}{2}\right)^{k}, \\
\mu_{S, R_{i}}=\sqrt{\frac{B \bar{\Gamma}_{S, R_{i}} / 2}{m_{S, R_{i}}+B \bar{\Gamma}_{S, R_{i}} / 2}},
\end{gathered}
$$

and

$$
P_{e / \Delta}^{S-D F}=A \iint Q(\sqrt{B(x+u)}) p_{\Gamma_{S, D}}(x) p_{\Gamma_{R_{s e l \Delta}, D}}(u) d x d u
$$

$\mathrm{R}_{\text {sel } \Delta}$ is the selected relay in set $\Delta$. This relay offers the highest SNR among relays that have correctly decoded :

$$
\Gamma_{R_{\text {sel }}, D}=\max _{i \in \Delta} \Gamma_{R_{i}, D}
$$

Therefore, the PDF of the SNR is given by

$$
\begin{aligned}
p_{\Gamma_{R_{s e l}, D}}(x) & =\sum_{i \in \Delta} p_{\Gamma_{R_{i}, D}}(x) \prod_{j \in \Delta, j \neq i} P_{\Gamma_{R_{j}, D}}(x), \\
P_{\Gamma_{R_{i}, D}}(u) & =1-\frac{G\left(m_{R_{i}, D}, \frac{m_{R_{i}, D}}{\bar{\Gamma}_{R_{i}, D}} u\right)}{\Gamma\left(m_{R_{i}, D}\right)},
\end{aligned}
$$

$\mathrm{G}(\mathrm{m}, \mathrm{u})$ is the incomplete gamma function, $\mathrm{p}_{\Gamma \mathrm{Ri}, \mathrm{D}}$ is written similarly to (5).

\section{AF with all participating relaying}

The SNR at D is the sum of SNRs from all N relays [21-22] since MRC combination is performed 


$$
\begin{gathered}
\Gamma_{D}=\Gamma_{S, D}+\sum_{k=1}^{N} \Gamma_{S, R_{k}, D} \\
\Gamma_{S, R_{k}, D}=\frac{\Gamma_{S, R_{k}} \Gamma_{R_{k}, D}}{1+\Gamma_{S, R_{k}}+\Gamma_{R_{k}, D}},
\end{gathered}
$$

In order to obtain simple equations, the following lower and upper bounds will be used

$$
\Gamma_{S, R_{k}, D}^{\text {low }}=\frac{1}{2} \min \left\{\Gamma_{S, R_{k}}, \Gamma_{R_{k}, D}\right\}<\Gamma_{S, R_{k}, D}<\Gamma_{S, R_{k}, D}^{u p}=\min \left\{\Gamma_{S, R_{k}}, \Gamma_{R_{k}, D}\right\}
$$

We have

$$
\Gamma_{D} \leq \Gamma_{D}^{u p}=\Gamma_{S, D}+\sum_{k=1}^{N} \Gamma_{S, R_{k}, D}^{u p}
$$

\subsection{Lower bound on SEP}

In the following, we derive a lower bound of the Symbol Error Probability (SEP) using the above upper bound on SNR. In next subsection, we explain how we can easily plot an upper bound of the SEP using the above lower bound on SNR. The MGF of SNR can be written as

$$
\mathrm{M}_{\Gamma_{D}^{u p}}(s)=\mathrm{M}_{\Gamma_{S, D}}(s) \prod_{k=1}^{N} \mathrm{M}_{\Gamma_{S, R_{k}, D}^{u p}}(s)
$$

where

$$
\mathrm{M}_{\Gamma_{S, D}}(s)=\left(1+s \frac{\bar{\Gamma}_{S, D}}{m_{S, D}}\right)^{-m_{S, D}}
$$

The CDF is equal to [11]

$$
\begin{array}{r}
F_{\Gamma_{S, R_{k}, D}^{u p}}(\gamma)=1-P\left(\Gamma_{S, R_{k}}>\gamma\right) P\left(\Gamma_{R_{k}, D}>\gamma\right) \\
=1-\frac{G\left(m_{S, R_{k}}, \frac{m_{S, R_{k}}}{\bar{\Gamma}_{S, R_{k}}}\right) G\left(m_{R_{k}, D}, \frac{m_{R_{k}, D}}{\bar{\Gamma}_{R_{k}, D}} \gamma\right)}{\left(m_{S, R_{k}}-1\right) !\left(m_{R_{k}, D}-1\right) !}
\end{array}
$$


where $G(.,$.$) is the upper incomplete gamma function$

$$
G(x, y)=\int_{y}^{\infty} e^{-t} t^{x-1} d t
$$

The PDF is obtained by a simple derivative of above equation

$$
\begin{aligned}
& p_{\Gamma_{S, R_{k}, D}^{u p}}(\gamma)=\left[\left(\frac{m_{S, R_{k}}}{\bar{\Gamma}_{S, R_{k}}}\right)^{m s, R_{k}} \gamma^{m s, R_{k}}-1 e^{\frac{m}{\Gamma_{S, R_{k}}} \gamma}\right. \\
& \times G\left(m_{R_{k}, D}, \frac{m_{R_{k}, D}}{\bar{\Gamma}_{R_{k}, D}} \gamma\right)+\left(\frac{m_{R_{k}, D}}{\bar{\Gamma}_{R_{k}, D}}\right)^{m_{R_{k}, D}} \gamma^{m_{R_{k}, D}-1} e^{\frac{m_{R_{k}, D}}{\bar{\Gamma}_{R_{k}, D}} \gamma} \\
& \left.\times G\left(m_{S, R_{k}}, \frac{m_{S, R_{k}}}{\bar{\Gamma}_{S, R_{k}}} \gamma\right)\right] \frac{1}{\left(m_{S, R_{k}}-1\right) !\left(m_{R_{k}, D}-1\right) !}
\end{aligned}
$$

A Laplace Transform (LT) of the PDF gives the MGF (Moment Generating Function) [19]

$$
\begin{aligned}
& M_{\Gamma_{s, R_{k}, D}^{u_{p}}}(s)=\left(\frac{m_{S, R_{k}}}{\bar{\Gamma}_{S, R_{k}}}\right)^{m_{s, R_{k}}}\left(\frac{m_{R_{k}, D}}{\bar{\Gamma}_{R_{k}, D}}\right)^{m_{R_{k}, D}} \frac{\left(m_{S, R_{k}}+m_{R_{k}, D}-1\right) !}{\left(m_{S, R_{k}}-1\right) !\left(m_{R_{k}, D}-1\right) !} \\
& {\left[\frac{1}{m_{S, R_{k}}}{ }_{2} F_{1}\left(1, m_{S, R_{k}}+m_{R_{k}, D} ; m_{S, R_{k}}+1 ; \frac{\frac{m_{s, R_{k}}}{\overline{\Gamma_{S, R_{k}}}}+s}{\frac{m_{S, R_{k}}}{\overline{\Gamma_{s, R_{k}}}}+\frac{m_{R_{k}, D}}{\overline{\Gamma_{R_{k}, D}}}+s}\right)+\right.} \\
& \left.\frac{1}{m_{R_{k}, D}}{ }_{2} F_{1}\left(1, m_{S, R_{k}}+m_{R_{k}, D} ; m_{R_{k}, D}+1 ; \frac{\frac{m_{R_{k}, D}}{\bar{\Gamma}_{R_{k}, D}}+s}{\frac{m_{S, R_{k}}}{\overline{\Gamma_{s, R_{k}}}}+\frac{m_{R_{k}, D}}{\bar{\Gamma}_{R_{k}, D}}+s}\right)\right] \\
& \times \frac{1}{\left(\frac{m s, R_{k}}{\bar{\Gamma}_{\mathcal{S}, R_{k}}}+\frac{m_{R_{k}, D}}{\overline{\bar{\Gamma}}_{R_{k}, D}}+s\right)^{m S, R_{k}+m_{R_{k}, D}}}
\end{aligned}
$$

${ }_{2} \mathrm{~F}_{1}(., .,$.$) is Gauss' hypergeomeric function.$

If the fading figure of the first hop is equal to that of the second hop and average SNR of first hop is equal to that of the second hop, we have

$$
\begin{array}{r}
m_{S, R_{k}}=m_{R_{k}, D}=m_{k} \text { and } \bar{\Gamma}_{S, R_{k}}=\bar{\Gamma}_{R_{k}, D}=\rho_{k} \\
M_{\Gamma_{S, R_{k}, D}^{u p}}(s)=\left(\frac{m_{k}}{\rho_{k}}\right)^{2 m_{k}} \frac{\left(2 m_{k}-1\right) !}{m_{k}\left(\left(m_{k}-1\right) !\right)^{2}} \\
\times \frac{2}{\left(\left(\frac{2 m_{k}}{\rho_{k}}\right)+s\right)^{2 m_{k}}{ }_{2} F_{1}\left(1,2 m_{k} ; m_{k}+1 ; \frac{\frac{m_{k}}{\rho_{k}}+s}{\frac{2 m_{k}}{\rho_{k}}+s}\right) .}
\end{array}
$$


Our results are valid for any channels and the above equation is provided for the case that the fading parameter of the first hop $m$ is equal to that of the second hop.

The Symbol Error Probability (SEP) for I-PSK modulation is equal to [19]

$$
P_{e_{D}}^{A F}=\frac{1}{\pi} \int_{0}^{(I-1) \pi / I} M_{\Gamma_{D}^{u p}}\left(\frac{g_{P S K}}{\sin ^{2}(\theta)}\right) d \theta
$$

where $g_{P S K} \doteq \sin ^{2} \pi / I$ and $M_{\Gamma_{D}^{u p}}$ is computed using (20), (21) and (25).

\subsection{Upper bound on SEP}

Let

$$
\begin{gathered}
Y=\Gamma_{S, R_{k}, D}^{l o w} \\
X=\Gamma_{S, R_{k}, D}^{u p}
\end{gathered}
$$

We have

$$
\mathrm{Y}=\mathrm{X} / 2
$$

Therefore, the CDF, PDF and MGF of Y can be deduced from the following simple equations

$$
\begin{aligned}
& P_{Y}(u)=P_{X}(2 u), \mathrm{CDF} \\
& p_{Y}(u)=2 p_{X}(2 u), \mathrm{PDF} \\
& M_{Y}(s)=E\left(e^{-s Y}\right)=M_{X}(s / 2), \mathrm{MGF}
\end{aligned}
$$

Having the MGF of relaying link, we can easily deduce an upper bound of the SEP using results of previous subsection.

\section{DF with all participating relaying}

Let $\theta$ be the set of relays that have correctly received the transmitted symbol by the source. It is assumed that all relays transmit over orthogonal channels. The symbol error probability at $\mathrm{D}$ is equal to

$$
P_{e_{D}}^{D F}=\sum_{\Theta} P_{e_{D} \mid \Theta}^{D F} P(\Theta)
$$




$$
P(\Theta)=\prod_{i \in \Theta}\left(1-P_{e_{R_{i}}}\right) \prod_{j \notin \Theta} P_{e_{R_{j}}} .
$$

$\mathrm{Pe}_{\mathrm{Rj}}$ is the SEP at relay $\mathrm{Rj}$.

\section{1 SEP at the relay}

The PDF of the SNR at Rk, is equal to

$$
p_{\Gamma_{S, R_{k}}}(\gamma)=f\left(\gamma, \frac{\bar{\Gamma}_{S, R_{k}}}{m_{S, R_{k}}}, m_{S, R_{k}}\right)
$$

where

$$
f(\gamma, a, l)=\frac{\gamma^{l-1}}{a^{l}(l-1) !} e^{-\frac{\gamma}{a}}, \gamma \geq 0
$$

The SEP at k-th relay is equal to

$$
\begin{aligned}
P_{e_{R_{k}}} & =A \int Q(\sqrt{B \gamma}) p_{\Gamma_{S, R_{k}}}(\gamma) d \gamma \\
& =\Psi\left(m_{S, R_{k}}, S, R_{k}\right)
\end{aligned}
$$

where $\mathrm{A}$ and $\mathrm{B}$ depend on the considered modulation (for example, $\mathrm{A}=1, \mathrm{~B}=2$ for BPSK),

$$
Q(x)=\frac{1}{\sqrt{2 \pi}} \int_{x}^{+\infty} e^{-\frac{u^{2}}{2}} d u
$$

and

$$
\Psi(l, X, Y)=A\left[\frac{1-\mu_{X, Y}}{2}\right]^{l} \sum_{i=0}^{l-1} C_{l+i-1}^{i}\left(\frac{1+\mu_{X, Y}}{2}\right)^{i}
$$




$$
\begin{gathered}
C_{n}^{k}=\frac{n !}{k !(n-k) !} \\
\mu_{X, Y}=\sqrt{\frac{B \bar{\Gamma}_{X, Y}}{2 m_{X, Y}+B \bar{\Gamma}_{X, Y}}}
\end{gathered}
$$

\subsection{Conditional SEP at the destination}

When the set of relays having correctly decoded is $\theta$, the conditional SEP at D is equal to

$$
P_{e_{D} \mid \Theta}^{D F}=A \int Q(\sqrt{B \gamma}) p_{\Gamma_{D \mid \Theta}}(\gamma) d \gamma
$$

where

$$
\Gamma_{D \mid \Theta}=\Gamma_{S, D}+\sum_{k \in \Theta} \Gamma_{R_{k}, D},
$$

To obtain the PDF, we can use the Moment Generating Function (MGF) of SNR. In fact, the MGF of sum of independent random variable is equal to the product of MGF :

$$
M_{\Gamma_{D \mid \Theta}}(s)=M_{\Gamma_{S, D}}(s) \prod_{k \in \Theta} M_{\Gamma_{R_{k}, D}}(s)
$$

where

$$
M_{\Gamma_{X, Y}}(s)=\left(1+s \frac{\bar{\Gamma}_{X, Y}}{m_{X, Y}}\right)^{-m_{X, Y}}
$$

A fraction decomposition gives

$$
\begin{aligned}
M_{\Gamma_{D \mid \Theta}}(s)= & \sum_{l=1}^{m_{S, D}} \frac{\alpha_{0, l}}{\left(1+\frac{\overline{\bar{\Gamma}}_{S, D}}{m_{S, D}} s\right)^{l}} \\
& +\sum_{k \in \Theta} \sum_{l=1}^{m_{R_{k}, D}} \frac{\alpha_{k, l}}{\left(1+\frac{\overline{\bar{\Gamma}}_{R_{k}, D}}{m_{R_{k}, D}} s\right)^{l}}
\end{aligned}
$$


where the residues are

$$
\begin{aligned}
\alpha_{0, l} & =\left(\frac{m_{S, D}}{\bar{\Gamma}_{S, D}}\right)^{m_{S, D}-l} \frac{1}{\left(m_{S, D}-l\right) !} \\
& \times\left.\left[\prod_{k \in \Theta}\left(1+\frac{\bar{\Gamma}_{R_{k}, D}}{m_{R_{k}, D}} s\right)^{-m_{R_{k}, D}}\right]^{\left(m_{S, D}-l\right)}\right|_{s=-\frac{m_{S, D}}{\bar{\Gamma}_{S, D}}},
\end{aligned}
$$

and

$$
\begin{aligned}
\alpha_{k, l}= & \frac{\left(\frac{m_{R_{k}, D}}{\bar{\Gamma}_{R_{k}, D}}\right)^{m_{R_{k}, D}-l}}{\left(m_{R_{k}, D}-l\right) !}\left[\left(1+s \frac{\bar{\Gamma}_{S, D}}{m_{S, D}}\right)^{-m_{S, D}}\right. \\
& \left.\times \prod_{\substack{i \in \Theta \\
i \neq k}}\left(1+\frac{\bar{\Gamma}_{R_{i}, D}}{m_{R_{i}, D}} s\right)^{-m_{R_{i}, D}}\right]\left.^{\left(m_{R_{k}, D}-l\right)}\right|_{s=-\frac{m_{R_{k}, D}}{\bar{\Gamma}_{R_{k}, D}}}
\end{aligned}
$$

$\mathrm{f}^{(\mathrm{n})}$ is the $\mathrm{n}$-th derivative of $\mathrm{f}$ and $\mathrm{g}(\mathrm{x}) \mid \mathrm{x}=\mathrm{c}=\mathrm{g}(\mathrm{c})$.

The PDF is deduce by making an inverse LT

$$
\begin{aligned}
p_{\Gamma / \Theta}(\gamma)= & \sum_{l=1}^{m_{S, D}} \alpha_{0, l} f\left(\gamma, \frac{\bar{\Gamma}_{S, D}}{m_{S, D}}, l\right) \\
& +\sum_{k \in \Theta} \sum_{j=1}^{m_{R_{k}, D}} \alpha_{k, j} f\left(\gamma, \frac{\bar{\Gamma}_{R_{k}, D}}{m_{R_{k}, D}}, j\right)
\end{aligned}
$$

By using previous equations, we have

$$
\begin{aligned}
P_{e_{D} / \Theta}^{D F}= & \sum_{l=1}^{m_{S, D}} \alpha_{0, l} \Psi(l, S, D) \\
& +\sum_{k \in \Theta} \sum_{j=1}^{m_{R_{k}, D}} \alpha_{k, j} \Psi\left(j, R_{k}, D\right)
\end{aligned}
$$

\section{SEP of cooperative AF relaying for cognitive radio networks}

The system model is shown in Fig. 2. In the primary network, there are primary transmitter and receiver denoted by PT and PR. In the secondary network, there are a secondary source S, secondary destination $\mathrm{D}$ and $\mathrm{N}$ relays Ri. Only relays that generate interference to PR lower 
than a predefined threshold $\mathrm{T}$ are activated, i.e.,

$$
I_{R_{i} P_{R}}=E_{i}\left|f_{R_{i} P_{R}}\right|^{2}<T \text {. }
$$

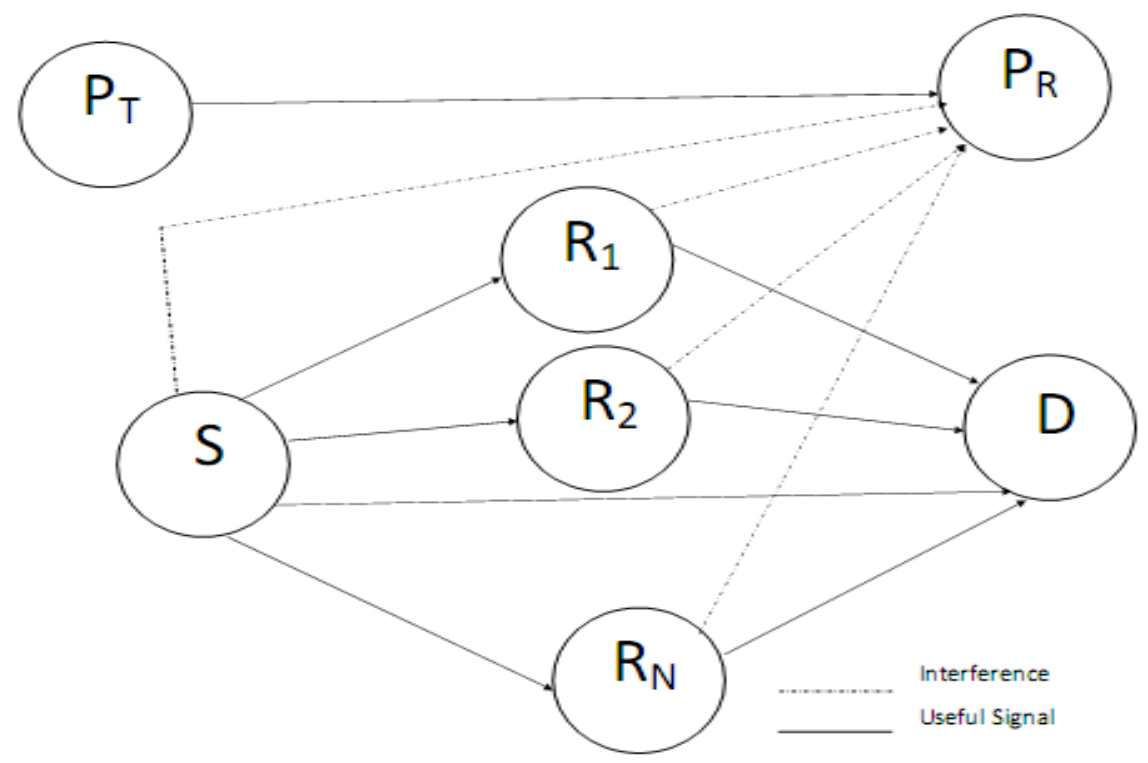

Fig. 2. System model for cognitive radio networks

For all participating relaying, all relays that verify interference constraint transmit over orthogonal channels.

For Selective AF, the best relay among those that verify interference constraints transmits.

Interference measurements are done at primary receiver PR. Then, the PR sends some signalization to central node to inform it about candidate relays (i.e. those that generate interference to PR lower than T). The CSI of first and second hop of these candidate relays are sent to the central node. Finally, the central node activates the candidate relay that verifies interference constraints and offers the highest end-to-end SNR (3).

The SEP is written as

$$
P_{e_{D}}^{A F}=\sum_{\Theta} P_{e_{D} \mid \Theta}^{A F} P(\Theta)
$$

$\mathrm{P}_{\mathrm{ed}}{ }^{\mathrm{AF}} \mid \theta$ is given in equations (4) and (27) respectively for selective AF and all participating AF. We have only to replace the set of available relays $(1, \ldots, \mathrm{N})$ by $\theta, \mathrm{P}(\theta)$ is the probability that only relays in set $\theta$ verify interference constraints

$$
P(\Theta)=\prod_{i \in \Theta} P\left(I_{R_{i} P_{R}}<T\right) \prod_{j \notin \Theta} P\left(I_{R_{j} P_{R}}>T\right) .
$$


where

$$
P\left(I_{R_{i} P_{R}}<T\right)=1-\frac{G\left(m_{R_{i} P_{R}}, \frac{m_{R_{i} P_{R}} T}{\overline{I_{R_{i}, P_{R}}}}\right)}{\Gamma\left(m_{R_{i}, P_{R}}\right)}
$$

where

$$
\overline{I_{R_{i} P_{R}}}=E\left(I_{R_{i} P_{R}}\right)=\frac{E_{i}}{d_{R_{i} P_{R}}^{\beta}}
$$

is the average interference that depends on the distance between Ri and PR, $d_{R i P R}, \beta$ is the path loss exponent.

\section{SEP of cooperative DF relaying for cognitive radio networks}

The system model is shown in Fig. 2. Only relays that have correctly decoded the symbol and generate interference to PR lower than a predefined threshold $\mathrm{T}$ are activated.

For all participating relaying, all relays that verify interference constraint and having correctly decoded, transmit over orthogonal channels.

For Selective DF, the best relay among those that verify interference constraints and having correctly decoded transmits. The PR sends some signalization to central node to inform it about candidate relays (i.e. those that generate interference to PR lower than T). The relays that have correctly decoded send also some signalization to inform the central node about it. The destination sends also the CSI of second hop of different relays. Finally, the central node activates only the relay that has correctly decoded, verifies interference constraints and offers the highest SNR of second hop.

The SEP is written as

$$
P_{e_{D}}^{D F}=\sum_{\Theta} P_{e_{D} \mid \Theta}^{D F} P(\Theta) .
$$

where $\mathrm{P}^{\mathrm{DF}}{ }_{\text {eD }} \mid \theta$ is given in equations (12) and (36) respectively for selective $\mathrm{DF}$ and all participating DF. We have only to replace the set of available relays $(1, \ldots, N)$ by $\theta, P(\theta)$ is the probability that only relays in set $\theta$ verify interference constraints and have correctly decoded the symbol

$$
P(\Theta)=\prod_{i \in \Theta} P\left(I_{R_{i} P_{R}}<T\right)\left(1-P e_{R_{i}}\right) \prod_{j \notin \Theta}\left[1-P\left(I_{R_{j} P_{R}}<T\right)\left(1-P e_{R_{j}}\right)\right]
$$

\section{Asymptotic analysis and Diversity-Multiplexing Tradeoff}

\subsection{Asymptotic analysis of all participating DF}

The CDF of the SNR of the Rk-D link is equal to 


$$
F_{\Gamma_{S R_{k}}}(x)=1-\frac{G\left(m_{R_{k} D}, \frac{m_{R_{k} D}}{\bar{\Gamma}_{R_{k} D}}\right)}{\left(m_{R_{k} D}-1\right) !} .
$$

Series representation of the incomplete Gamma function gives

$$
\frac{G\left(m_{R_{k} D}, \frac{m_{R_{k} D}}{\bar{\Gamma}_{R_{k} D}}\right)}{\left(m_{R_{k} D}-1\right) !}=1-\frac{1}{\left(m_{R_{k} D}-1\right) !} \sum_{n=0}^{+\infty} \frac{(-1)^{n}\left[\frac{m_{R_{k} D}}{\bar{\Gamma}_{R_{k} D}}\right]^{m_{R_{k} D}+n}}{n !\left(m_{R_{k} D}+n\right)} .
$$

Using (50) and (51), we obtain an approximation of the CDF at high SNR by using the first term of (51) :

$$
F_{\Gamma_{S R_{k}}}(x) \approx \frac{1}{m_{R_{k} D}\left(m_{R_{k} D}-1\right) !}\left[\frac{m_{R_{k} D} x}{\bar{\Gamma}_{R_{k} D}}\right]^{m_{R_{k} D}} .
$$

For all participating DF relaying and at high SNR, all relays are active and the SNR is equal to

$$
\Gamma_{D}=\Gamma_{S D}+\sum_{k=1}^{N} \Gamma_{R_{k} D}
$$

We have to derive the CDF of the total SNR to deduce the diversity order. We begin by considering the case of a single relay. The CDF of the SNR is given by

$$
P_{\Gamma_{D}}(z)=\int_{0}^{z} P_{\Gamma_{S D}}(z-x) p_{\Gamma_{R_{1} D}}(x) d x
$$

Using (52) which is also valid for the direct link, we obtain

$$
P_{\Gamma_{D}}(z) \approx z^{m_{S D}+m_{R_{1} D}} \frac{\left[\frac{m_{R_{1} D}}{\bar{\Gamma}_{R_{1} D}}\right]^{m_{R_{1} D}}\left[\frac{m_{S D}}{\bar{\Gamma}_{S D}}\right]^{m_{S D}}}{\left(m_{S D}+m_{R_{1} D}\right) !}
$$

which shows that the diversity order is equal to $\mathrm{m}_{\mathrm{SD}}+\mathrm{m}_{\mathrm{R} 1 \mathrm{D}}$. In case of many relays, a similar approach shows that the diversity order is equal to

$$
G_{d}=m_{S D}+\sum_{k=1}^{N} m_{R_{k} D}
$$

\subsection{Opportunistic DF}

We first derive the statistic of 


$$
\Gamma_{\max }=\max \left(\Gamma_{R_{1} D}, \Gamma_{R_{2} D}\right) .
$$

Using (52), we obtain

$$
\begin{aligned}
P_{\Gamma_{\max }}(x) & =P_{\Gamma_{R_{1} D}}(x) P_{\Gamma_{R_{2} D}}(x) \\
& \approx \frac{1}{m_{R_{1} D}\left(m_{R_{1} D}-1\right) !}\left[\frac{m_{R_{1} D} x}{\bar{\Gamma}_{R_{1} D}}\right]^{m_{R_{1} D}} \frac{1}{m_{R_{2} D}\left(m_{R_{2} D}-1\right) !}\left[\frac{m_{R_{2} D} x}{\bar{\Gamma}_{R_{2} D}}\right]^{m_{R_{2} D}}
\end{aligned}
$$

At high SNR, all DF relays have correctly decoded and the SNR is equal to

$$
\Gamma_{D}=\Gamma_{S D}+\max \left(\Gamma_{R_{1} D}, \ldots, \Gamma_{R_{N} D}\right)
$$

Using the same steps as previous section, we easily show that the S-DF protocol offer the same diversity as all participating DF given in (56).

\subsection{All participating and opportunistic AF}

For AF relaying, the SNR is given by

$$
\Gamma_{S R D}=\frac{\Gamma_{S R} \Gamma_{R D}}{1+\Gamma_{S R}+\Gamma_{R D}}<\min \left(\Gamma_{S R}, \Gamma_{R D}\right)
$$

At high SNR, the upper bound is very tight and the CDF can be approximated by

$$
P_{\Gamma_{S R D}}(x) \approx 1-P\left(\Gamma_{S R}>x\right) P\left(\Gamma_{R D}>x\right)
$$

Series representation of incomplete Gamma function gives

$$
P_{\Gamma_{S R D}}(x) \approx I_{1}+I_{2}-I_{1} I_{2}
$$

where

$$
\begin{aligned}
& I_{1}=\frac{1}{\left(m_{R D}-1\right) !} \sum_{n=0}^{+\infty} \frac{(-1)^{n}\left[\frac{m_{R D} x}{\bar{\Gamma}_{R D}}\right]^{m_{R D}+n}}{n !\left(m_{R D}+n\right)} . \\
& I_{2}=\frac{1}{\left(m_{S R}-1\right) !} \sum_{n=0}^{+\infty} \frac{(-1)^{n}\left[\frac{m_{S R} x}{\bar{\Gamma}_{S R}}\right]^{m_{S R}+n}}{n !\left(m_{S R}+n\right)} .
\end{aligned}
$$

At high SNR, the CDF can be approximated by the term corresponding to the lowest power of $\mathrm{X}$ : 


$$
P_{\Gamma_{S R D}}(x) \approx \frac{1}{m(m-1) !}\left[\frac{m x}{\bar{\Gamma}}\right]^{m}
$$

where

$$
\begin{aligned}
& m=\min \left(m_{S R}, m_{R D}\right) \\
& \bar{\Gamma}=\left\{\begin{array}{l}
\bar{\Gamma}_{S R} i f m_{S R}<m_{R D} \\
\bar{\Gamma}_{R D} i f m_{R D}<m_{S R}
\end{array}\right.
\end{aligned}
$$

Using the same steps as previous sections, we easily show that the diversity order of all participating $\mathrm{AF}$ and selective $\mathrm{AF}$ are equal to

$$
G_{d}=m_{S D}+\sum_{k=1}^{N} \min \left(m_{S R_{k}}, m_{R_{k} D}\right)
$$

\subsection{Diversity multiplexing tradeoff for Non cognitive network}

At high SNRs, the outage probability can be approximated by

$$
P_{\text {out }}=a\left(\frac{\gamma_{t h}}{\bar{\gamma}}\right)^{G_{d}}
$$

where $\mathrm{a}$ is a constant, $\gamma_{\mathrm{th}}$ is the outage threshold,

$$
\bar{\gamma}=\frac{E_{s}}{N_{0}}
$$

is the average SNR, Es is the transmitted energy per symbol, $\mathrm{N}_{0}$ is the noise power spectral density (PSD).

The outage threshold $\gamma_{\text {th }}$ is related in terms of the spectral efficiency $\mathrm{R}$ as follows [1]

$$
\gamma_{t h}=2^{2 R}-1
$$

for opportunistic relaying since we have two transmitter S and selected relay.

$$
\gamma_{t h}=2^{(N+1) R}-1
$$


for all participating relaying since we have $\mathrm{N}+1$ transmitters [1].

In addition, from the definition of [1], the spectral efficiency $R$ can be written with respect to the normalized spectral efficiency $r$ as

$$
R=r \log _{2}(1+\bar{\gamma})
$$

Therefore, we can write

$$
\gamma_{t h}=(1+\bar{\gamma})^{2 r}-1
$$

for opportunistic relaying and

$$
\gamma_{t h}=(1+\bar{\gamma})^{(N+1) r}-1
$$

for all participating relaying.

The diversity multiplexing tradeoff can be formulated as

$$
d(r)=\lim _{\bar{\gamma} \rightarrow \infty} \frac{\log P_{\text {out }}(\bar{\gamma}, r)}{\log \bar{\gamma}}
$$

Using equations (69)-(76), the diversity multiplexing tradeoff is written as

$$
d(r)=G_{d}(1-2 r)
$$

for opportunistic relaying.

$$
d(r)=G_{d}(1-(N+1) r)
$$

for all participating relaying.

The maximum diversity Gd defined in (56)-(68) is reached for $r=0$.

The diversity order of DF-Distributed Space Time coding (DSTC) is the same as all participating DF and opportunistic DF [2]. It is well known that DSTC achieves full diversity [2]. In DSTC, all relays transmit over the same channel [2]. Two orthogonal channels are required : one for the source and one for relays' transmission. Therefore, the DMT of DF-DSTC is similar to that of opportunistic DF : same diversity and same number of orthgonal channels required (i.e. 2).

\subsection{Diversity multiplexing tradeoff for cognitive network}

The diversity order depend on the set of relays that verify interferenc constraints. When the set of available relays that verify interfference constraints is $\theta$, the diversity order is equal to

$$
G_{d}(\theta)=m_{S D}+\sum_{k \in \theta} \min \left(m_{S R_{k}}, m_{R_{k} D}\right)
$$

for all participating AF and opportunistic AF. 


$$
G_{d}(\theta)=m_{S D}+\sum_{k \in \theta} m_{R_{k} D}
$$

for all participating and opportunistic DF.

Using the results of previous section, the diversity multiplexing tradeoff is written as

$$
d(r)=\sum_{\theta} G_{d}(\theta) p(\theta)(1-2 r)
$$

for opportunistic relaying.

$$
d(r)=\sum_{\theta} G_{d}(\theta) p(\theta)(1-(N+1) r)
$$

for all participating relaying. $\mathrm{p}(\theta)$ is defined in (46) and (49) respectiverly for AF and DF.

\section{Theoretical and simulation results}

This section provides some numerical and simulation results of both AF and DF protocols for BPSK modulation. Fig. 3-5 corresponds to relay selection with AF and DF relaying. Fig. 6-8 correspond to all participating relaying. We have allocated the same transmitted energy per symbol to the source and the selected relay, Ei=Es/2 where Es is the transmitted energy per usefull symbol for relay selection. $\mathrm{Ei}=\mathrm{Es} /(\mathrm{N}+1)$ for all participating relaying. We have used the same fading figure in all links denoted by $\mathrm{m}$. The distance between all nodes is equal to one but our results are valid for any network and channel configuration. We can model the power of channel coefficient as being proportional to $1 / \mathrm{d}^{\beta}$ where $\beta$ is the path loss exponent.

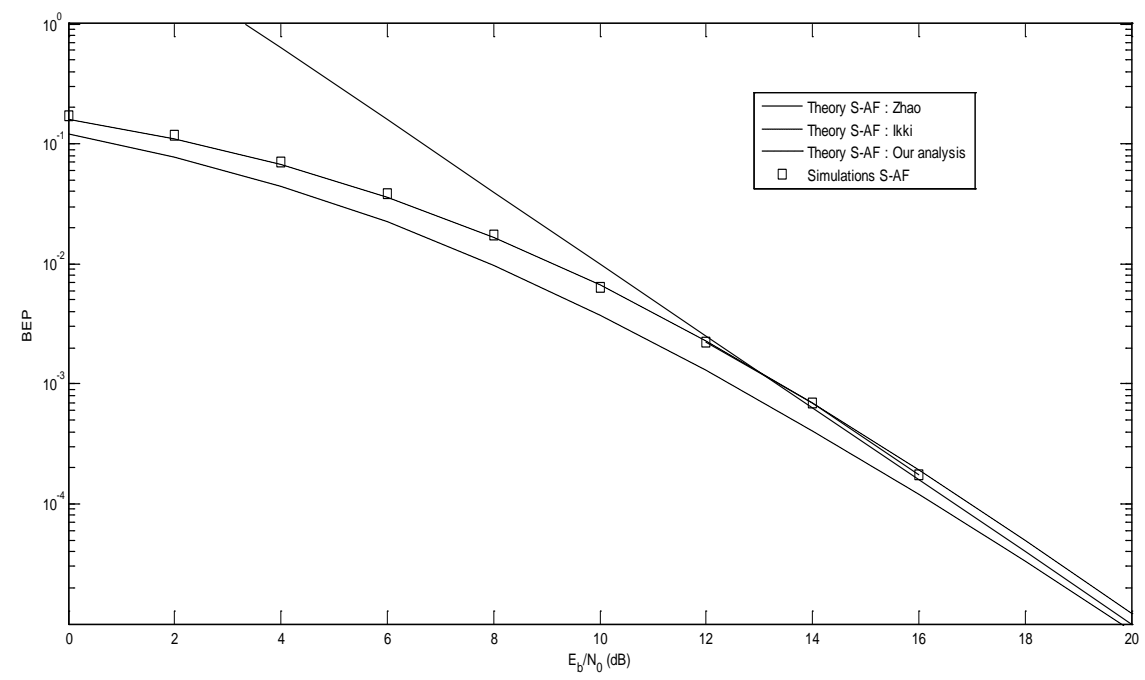

Fig. 3. BEP of Cooperative Selective Amplify and Forward protocol for Rayleigh fading channels

Fig. 3 shows the BEP of S-AF protocol for Rayleigh fading channel $(m=1)$. We have plotted the theoretical BEP as derived by Zhao [7] (equation (7), Ikki [8] (equation (14)) and the proposed study (equation (4)). As expected, the proposed study is in perfect accordance with the simulation results. 


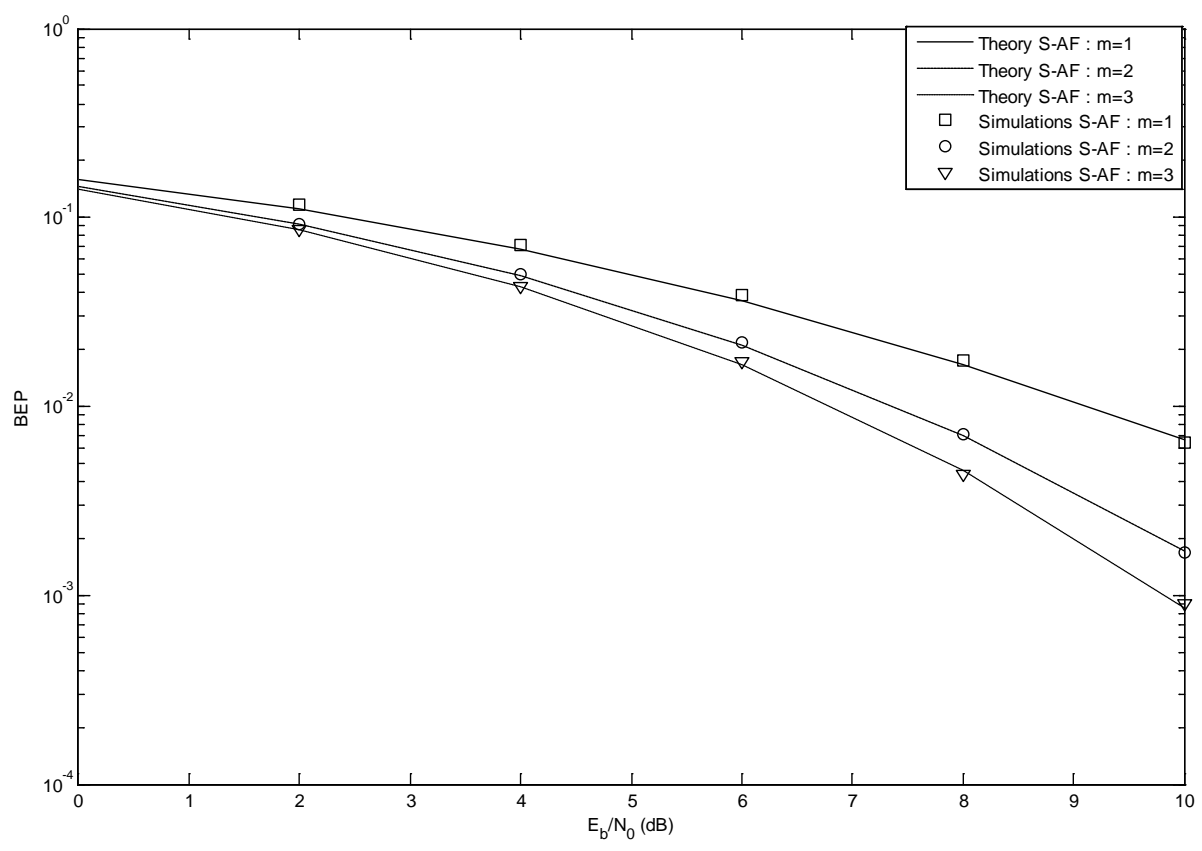

Fig. 4. BEP of Cooperative Selective Amplify and Forward protocol for Nakagami-m fading channels

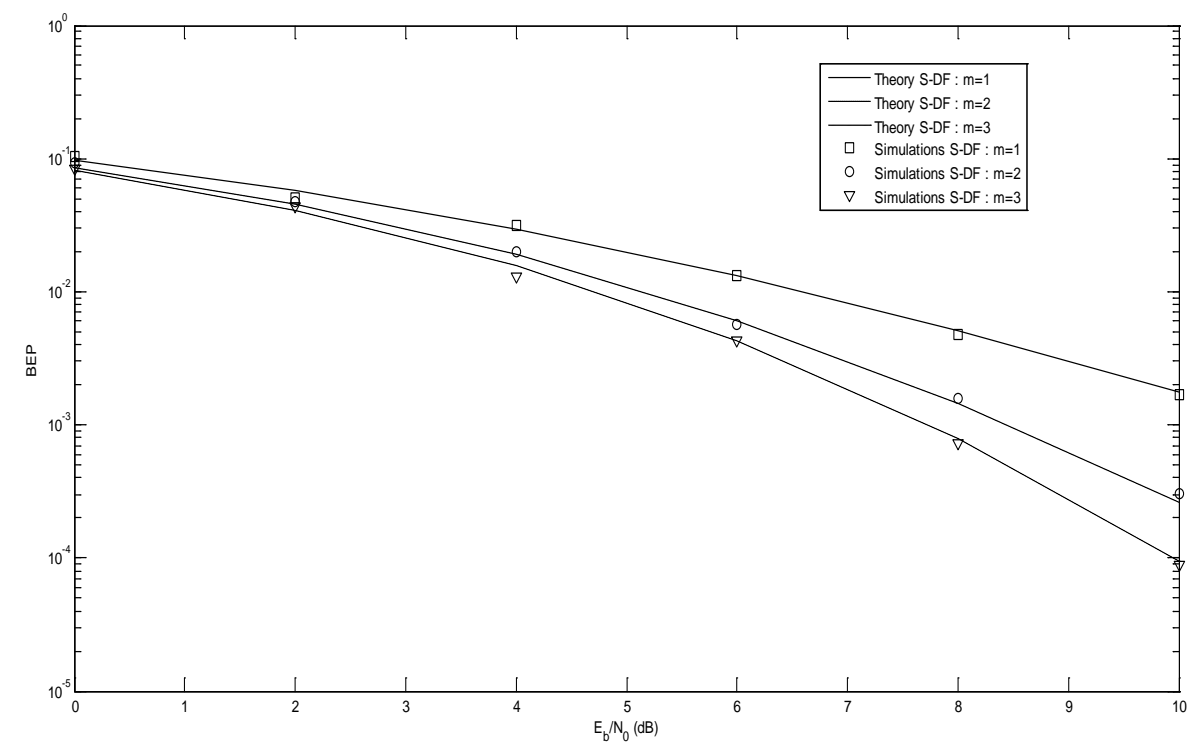

Fig. 5. BEP of Cooperative Selective Decode and Forward protocol for Nakagami-m fading channels

Fig. 4 and Fig. 5 show respectively the BEP of S-AF and S-DF protocols for Nakagami-m fading channels for $m=1,2$ and 3 . We notice that the simulation results are in accordance with the derived theoretical BEP. We also verify that the performance improves as $\mathrm{m}$ increases and that S-DF offers better performance than S-AF. 


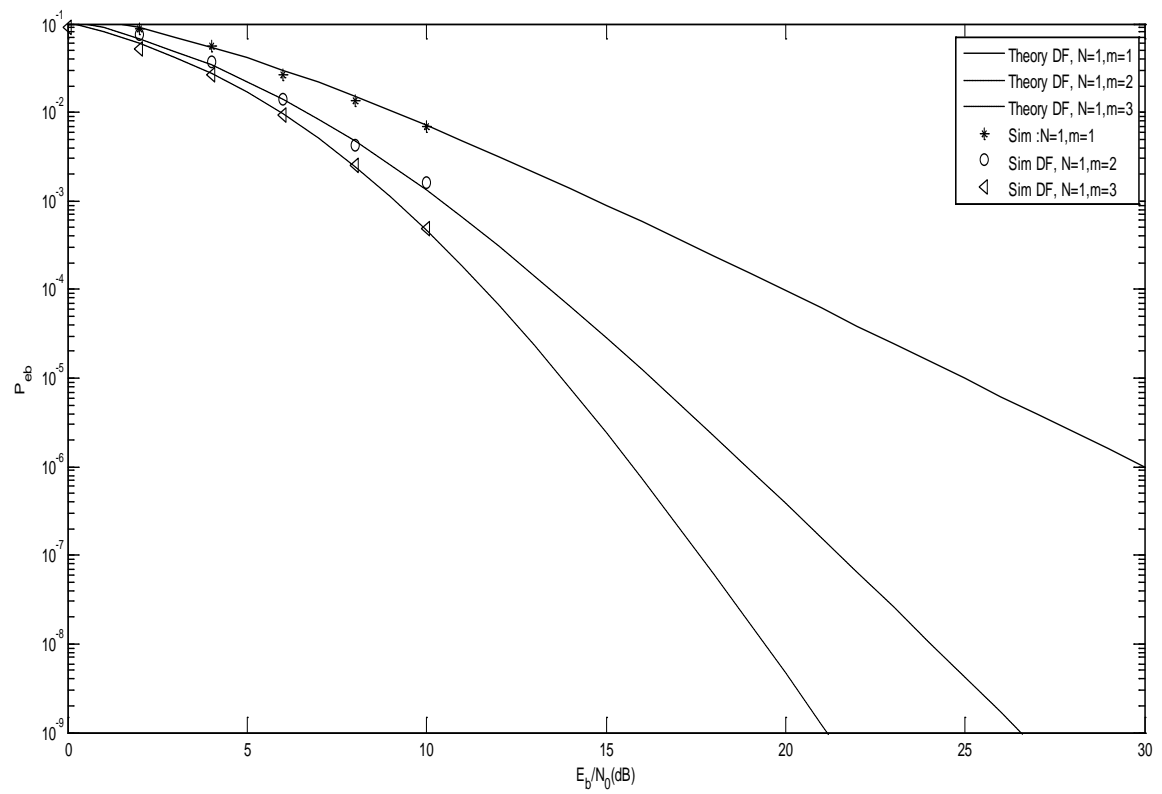

Fig. 6. BEP of Cooperative DF relaying for Nakagami-m fading channels

Fig. 6 shows the BEP of all particiapting DF relaying for BPSK modulation in the presence of a single relay $\mathrm{N}=1$ and different values of fading parameter $\mathrm{m}=1,2,3$. We notice that the performance improves as $\mathrm{m}$ increases. Simulation results agree well with theoretical ones.

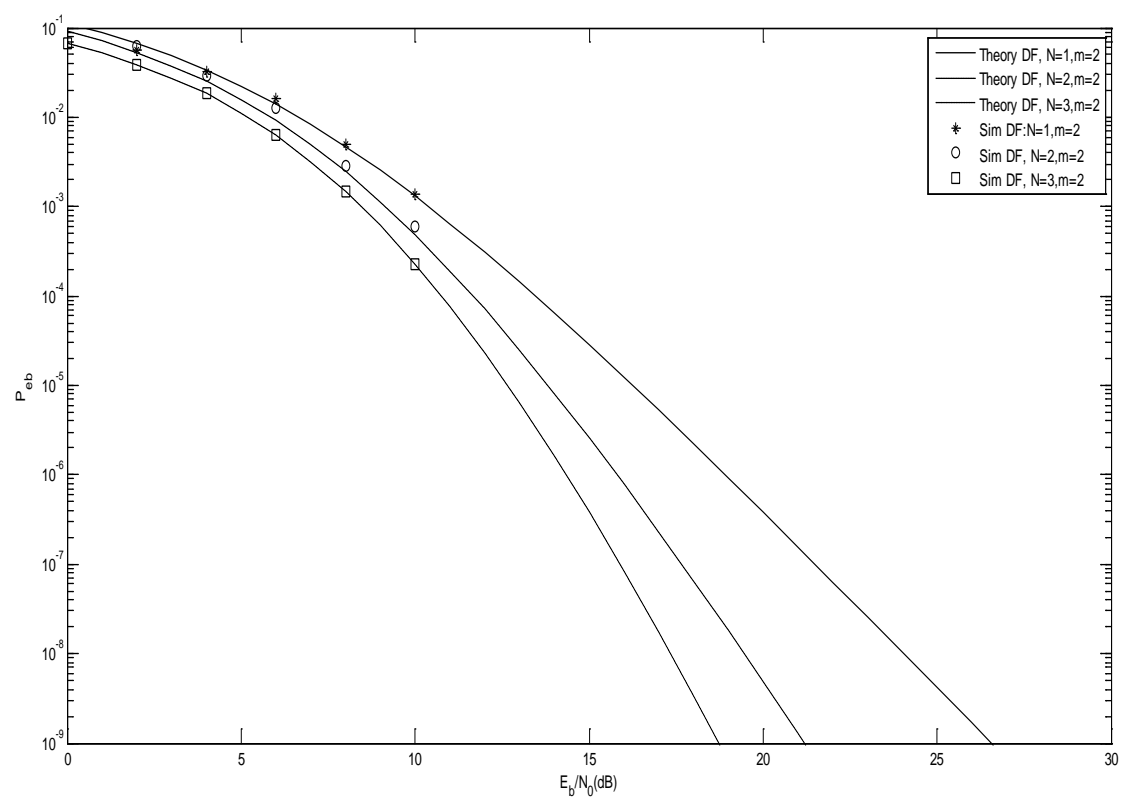

Fig. 7. BEP of Cooperative DF relaying for Nakagami-m fading channels : different number of relays and $\mathrm{m}=2$ 
Fig. 7 shows the results for $m=2$ and different number of all participating DF relays $N=1,2$ and 3. As the number of relays increases as the BEP decreases due to cooperative diversity. The signal is received through different relays and an MRC (Maximum Ratio Combination) of signals is performed at Destination.

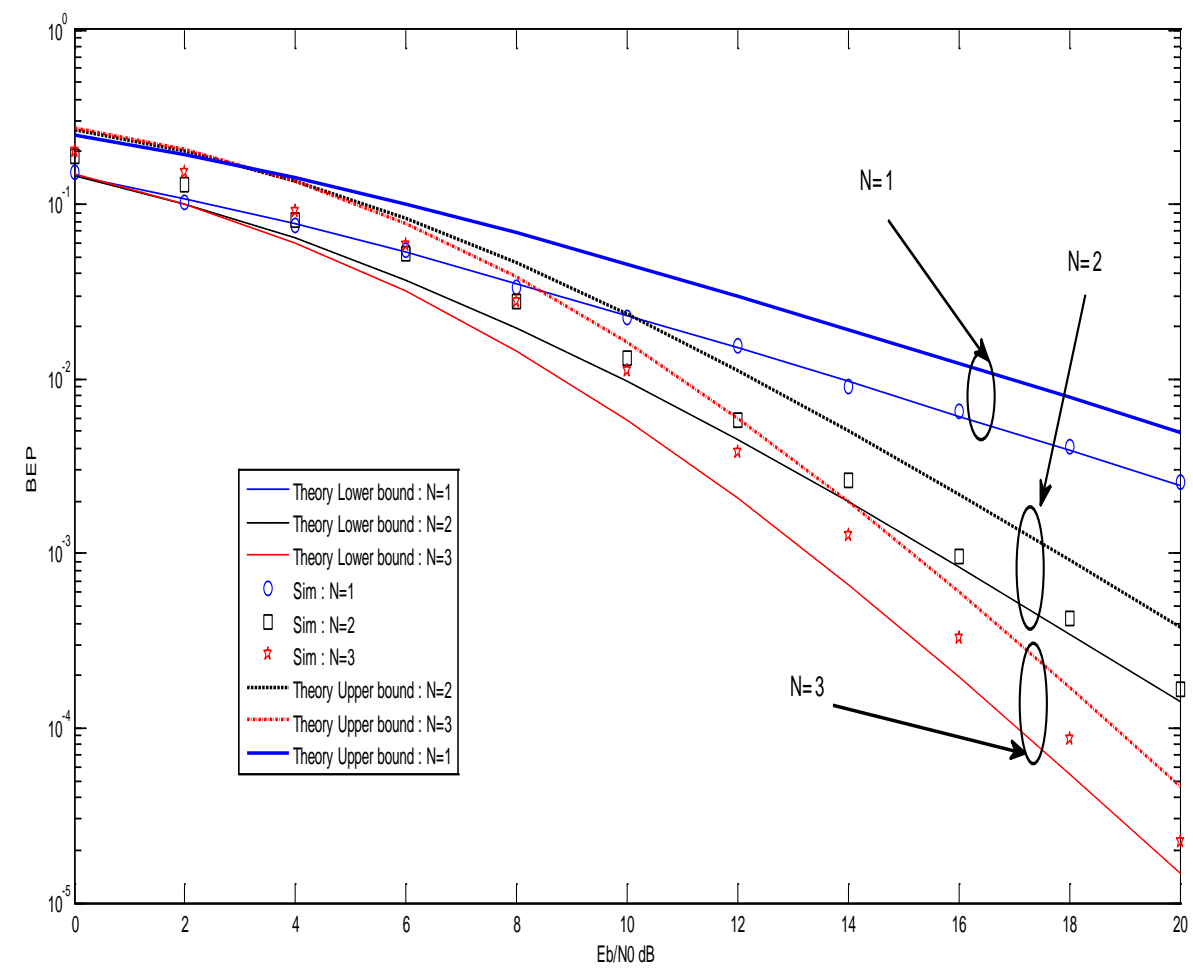

Fig. 8. BEP of Cooperative AF relaying for Nakagami-m fading channels : different number of relays and $\mathrm{m}=2$.

Fig. 8 shows the BEP for all participating AF relaying and $\mathrm{N}=1,2,3$ with $\mathrm{m}=2$ (fading parameter of Nakagami channel). The simulation results are between the lower and upper bound of BEP. It is well known that the upper bound of SNR (see equation 18 ) is very accurate at high SNR. 


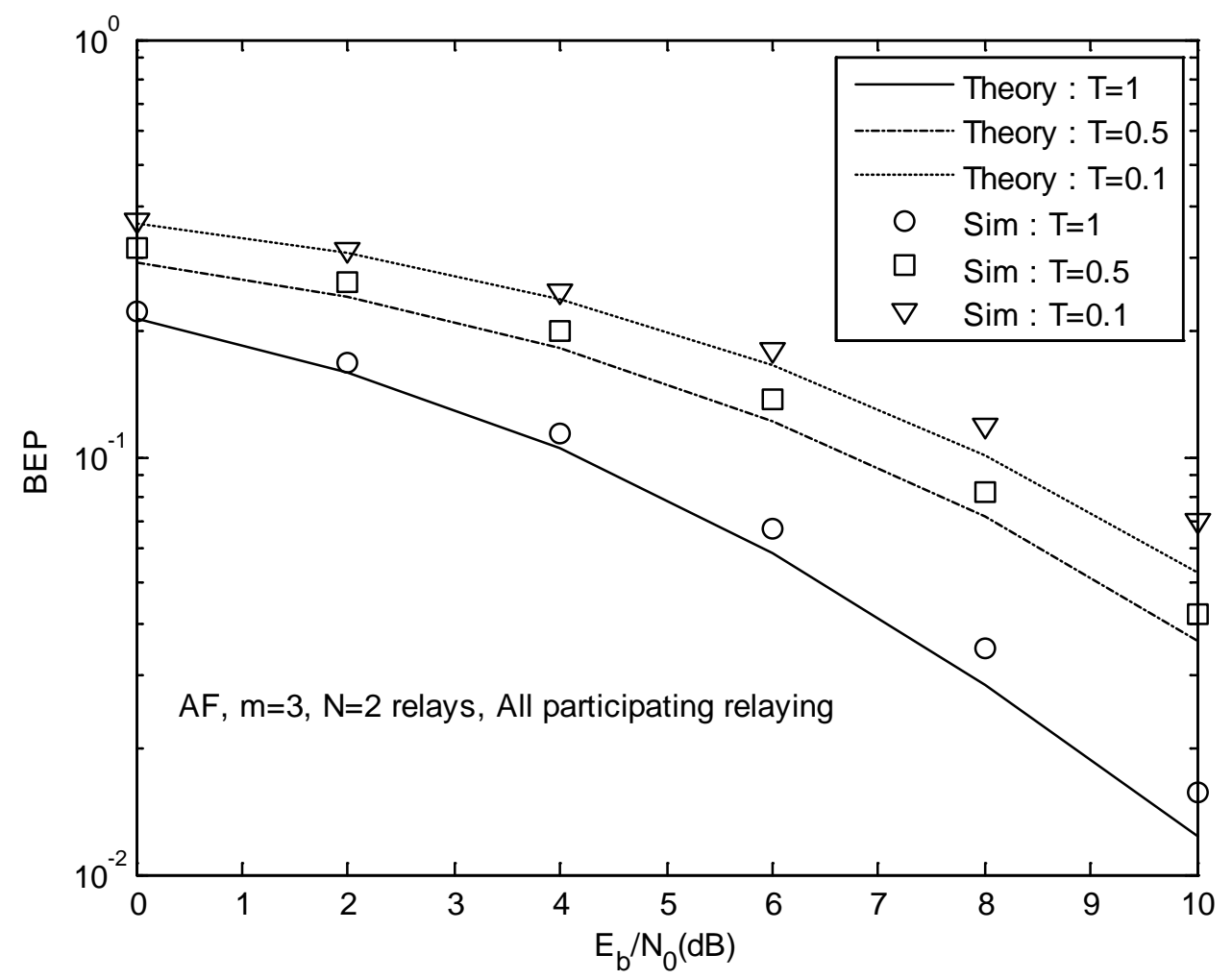

Fig. 9. BEP of all participating AF relaying : $m=3$ and $N=2$ relays for cognitive radio networks.

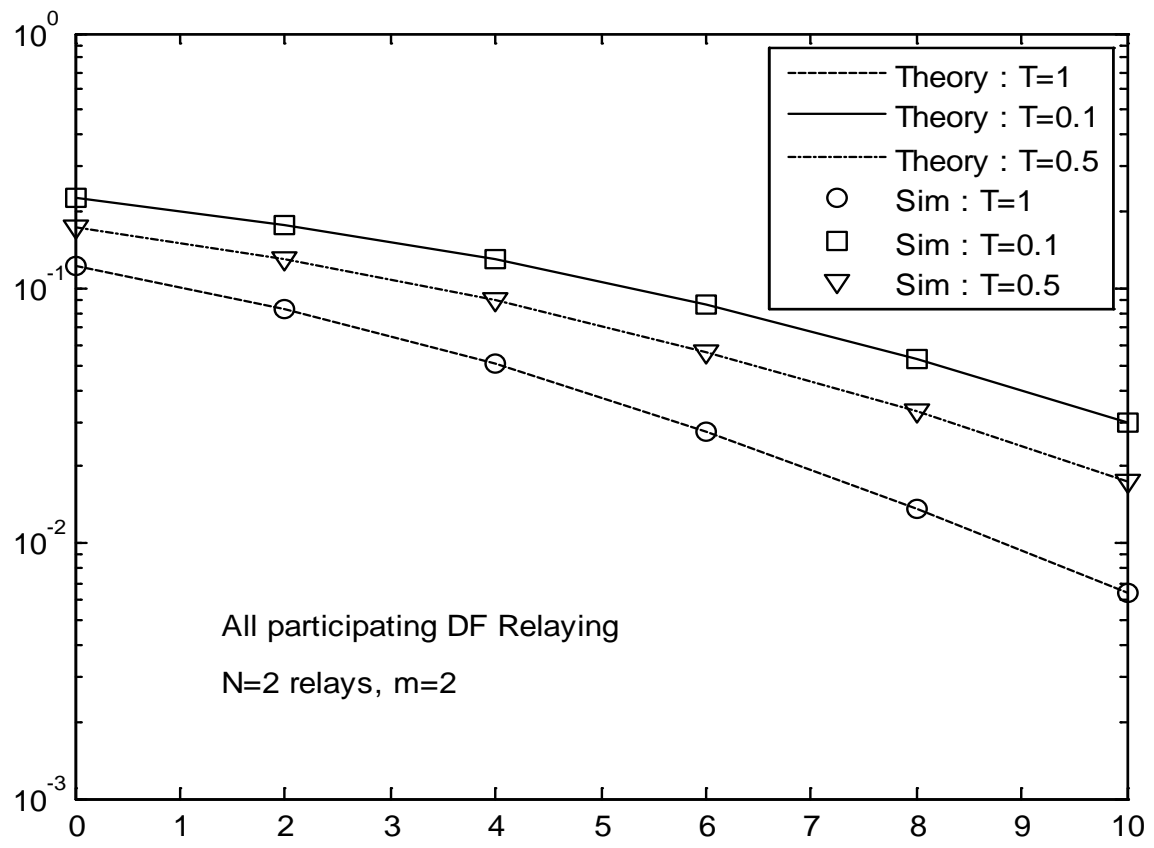

Fig. 10. BEP of all participating DF relaying : $m=2$ and $N=2$ relays for cognitive radio networks. 
Fig. 9 and Fig. 10 show respectively the BEP of all participating AF and DF relaying for cognitive radio networks. The distance between relays and primary receiver PR is equal to one. If this distance decreases (resp. increases), the performance degrades (resp. improves) since there are less (resp. more) available relays. We notice that the performance improves as the interference $\mathrm{T}$ threshold increases due to cooperative diversity since there are more available relays.

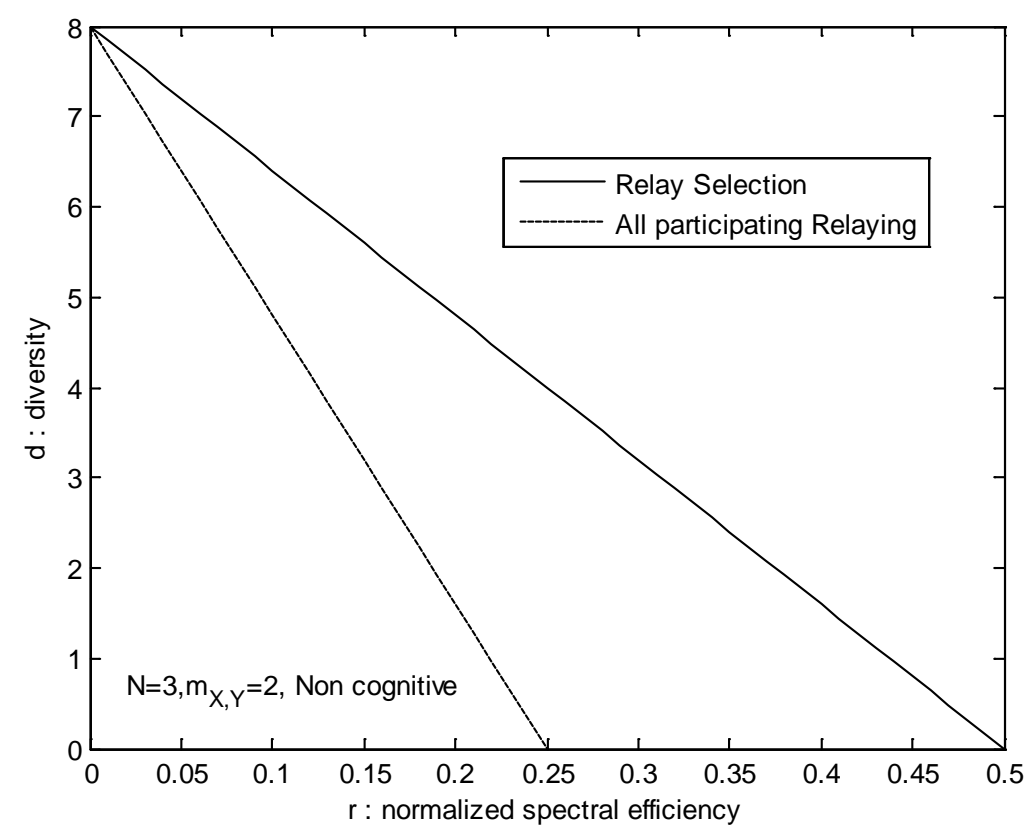

Fig. 11. DMT for non cogntive radio networks.

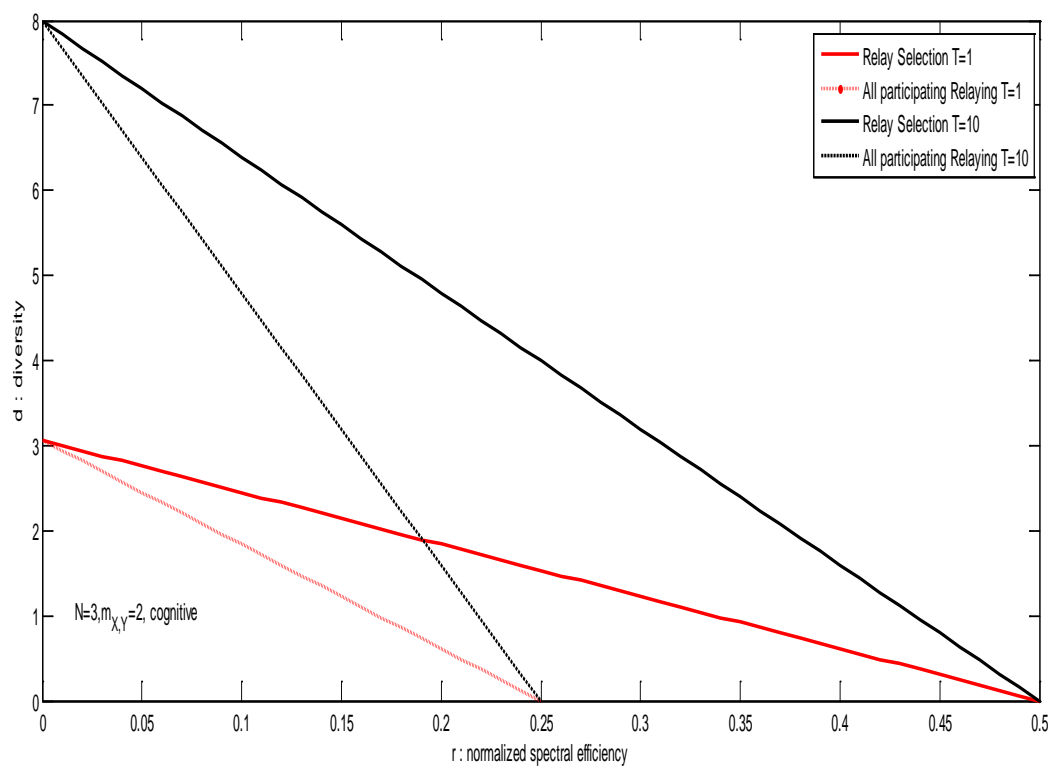

Fig. 12. DMT for cogntive radio networks. 
Fig. 11 and Fig. 12 show the DMT respectively for non cognitive and cognitive radio networks. There are $\mathrm{N}=3$ relays and $\mathrm{m}=2$ (fading parameter of Nakagami channel). Since the fading parameter of first and second hop is 2 for all links, the diversity order of AF is the same as DF (see (56) and (68)). Therefore, the DMT shown in Fig. 11 and Fig. 12 are valid for both AF and DF. In Fig. 11, the maximum diversity $(\mathrm{N}+1) * \mathrm{~m}=8$ is reached for normalized spectral efficiency $r=0$. The DMT of relay selection is better than all participating relaying. In Fig. 12, the diversity order reached for $\mathrm{r}=0$ depends on the interference threshold $\mathrm{T}$. The distance between secondary nodes and primary receiver is equal to one. For example, for $T=1$, the diversity is lower than $(\mathrm{N}+1) * \mathrm{~m}=8$ because relays are not always available and some of them generate an interference larger than $T$. However, for $T=10$, the obtained diversity is equal to 8 for $\mathrm{r}=0$ and almost all relays are available since the interference threshold $\mathrm{T}$ is large.

\section{Conclusion and perspectives}

In this paper, we have derived the exact and approximate SEP of cooperative systems using AF or DF relaying with best relay selection for Nakagami-m fading channels where the different links are assumed to be i.ni.d. We have verified that the derived SEP expressions are in accordance with the simulation results. In the second part of the paper, we studied the performance when all relays participate using AF and DF relaying. Results of DF relaying are exact and we have presented a lower and upper bounds of the SEP when AF relaying is used. Finally, in the last part of the paper, we have extended our results to cognitive radio networks where there is interference constraints : only relays that generate interference to primary receiver lower than a predefined threshold $\mathrm{T}$ can transmit. Both AF and DF protocols with and without relay selection were considered. As a perspective, we can study AF relaying with blind relays that don't need to estimate the channel. Also, it will be interesting to study Distributed Space Time Coding (DSTC) with AF relaying and derive its DMT.

\section{References}

[1] J. N. Laneman, D. N. C. Tse and G. W. Wornell, "Cooperative diversity in wireless networks : efficient protocol and outage behavior,” IEEE Transactions on Information Theory, Vol. 50, no 12, pp. 3062-3080, Oct 2004. Article (CrossRef Link)

[2] J. N. Laneman and G. W. Wornell, "Distributed space-time coded protocols for exploiting cooperative diversity in wireless networks," IEEE Transactions on Information Theory, Vol. 49, no 10, pp. 2415-2525, Oct 2003. Article (CrossRef Link)

[3] A. Sendoranis, E. Erkip and B. Aazhang, "User cooperation diversity, part I: System description,” IEEE Trans. Commun., Vol. 51, no 11, pp. 1927-1938, Nov. 2003. Article (CrossRef Link)

[4] A. Sendoranis, E. Erkip and B. Aazhang, "User cooperation diversity, part II: Implementation aspects and performance analysis,” IEEE Trans. Commun., Vol. 51, no 11, pp. 1939-1948, Nov. 2003. Article (CrossRef Link)

[5] P. A. Anghel, M. Kaveh, "Exact symbol error probability of a cooperative network in a Rayleigh fading environnement," IEEE Trans. on Wireless Commun., Vol. 3, no 5, pp. 1416-1421, Sept. 2004. Article (CrossRef Link)

[6] A. K. Sadek, W. Su and K. J. Ray Liu, "Multinode Cooperative communications in wireless Networks,” IEEE Trans. on Signal Processing, Vol. 55, No 1, pp. 341-355, Jan. 2007. Article (CrossRef Link)

[7] Y. Zhao, R. Adve, T. J. Lim, "Symbol Error Rate of Selection Amplify and Forward Relay Systems,” IEEE Communications Letters, Vol. 10, No. 11, pp. 757-759, Nov. 2006. Article (CrossRef Link) 
[8] S. S. Ikki, M. H. Ahmed, "Performance of Multiple-Relay Cooperative Diversity Systems with best Relay selection over Rayleigh fading channels," EURASIP Jounral on Advances in Signal Processing, Vol. 2008, 2008. Article (CrossRef Link)

[9] E. Beres, R. Adve, "Selection Cooperation in Multi-Source Cooperative Networks," IEEE Trans. on Wireless Commun., Vol. 7, No. 1, pp. 118-127, Jan. 2008. Article (CrossRef Link)

[10] T. A. Tsiftsis, G. K. Karagiannidis, P. T. Mathiopoulos, S. A. Kostopoulos, "Nonregenerative Dual-Hop Cooperative links with Selection Diversity,” EURASIP Journal on Wireless Communications and Networking, Vol. 2006, pp. 1-8, 2006. Article (CrossRef Link)

[11] J. N. Laneman and G. Wornell, "Energy-efficient antenna sharing and relaying for wireless networks," in Proc. of Wireless Commun. Networking Conf., vol. 1, pp. 3062-3080, 2000. Article (CrossRef Link)

[12] J. N. Laneman and G. Wornell, "Distributed space-time coded protocols for exploiting cooperative diversity in wireless networks," in Proc. of Wireless Commun. Networking Conf., vol. 1, pp. 77-81, 2002. Article (CrossRef Link)

[13] N.C. Beaulieu and J. Hu, "A closed-form expression for the outage probability of decode-and-forward relaying in dissimilar Rayleigh fading channels,” IEEE Commun. Lett., vol. 10, pp. 813-815, Dec. 2006. Article (CrossRef Link)

[14] In-Ho Lee and D. Kim, "BER analysis for decode-and-forward relaying in dissimilar Rayleigh fading channels,” IEEE Commun. Lett., vol. 11, pp. 52-54, Jan. 2007. Article (CrossRef Link)

[15] H. Boujemaa, "Exact and asymptotic BEP of cooperative DS-CDMA systems using Decode and Forward Relaying in the presence of multipath propagation," In press, IEEE Trans. on Wireless Commun., 2009. Year: 2009, Vol. 8, No. 9, Pages: 4464 - 4469, Article (CrossRef Link)

[16] H. Boujemaa, "Delays Analysis of Cooperative Truncated HARQ with Opportunistic Relaying,” IEEE Trans. on Vehicular Technology, Vol. 58, No. 9, 2009, pp. 4795 - 4804, Article (CrossRef Link)

[17] Diomidis S. Michalopoulos and George K. Karagiannidis and George S. Tombras, "Symbol error probability of decode and forward cooperative diversity in Nakagami-m fading channels,” Journal of the Franklin Institute, vol. 345, no. 7, pp. 723 - 728, 2008. Article (CrossRef Link)

[18] Boujemaa, H., Soussi, S. and Siala, M., "Evaluating the Performance of ARQ and HARQ I Over Nakagami Fading Channels,” Information and Communication Technologies ICTTA '06. 2nd, Vol. 2,pp. 2669 - 2673, 2006. Article (CrossRef Link)

[19] Marvin K. Simon and Mohamed-Slim Alouini, "Digital Communication over Fading Channels,” New York: Wiley, 2000. Article (CrossRef Link)

[20]Ikki, S. Ahmed, M.H, "Performance Analysis of Cooperative Diversity Wireless Networks over Nakagami-m Fading Channel,” IEEE Communications Letters, Vol. 11, No. 4, pp. 334-336, April 2007. Article (CrossRef Link)

[21]G. K. Karagiannidis, T. A. Tsiftsis, and R. K. Mallik, "Bounds of multihop relayed communications in Nakagami-m fading,” IEEE Trans. Commun., vol. 54, pp. 18-22, 2006. Article (CrossRef Link)

[22] M. Hasna and Alouini, "Harmonic mean and end-to-end performance of transmission systems with relays,” IEEE Trans. Commun., vol. 52, no. 1, 2004. Article (CrossRef Link)

[23]Trung Q. Duong; Daniel Benevides da Costa; Maged Elkashlan; Vo Nguyen Quoc Bao, "Cognitive Amplify-and-Forward Relay Networks Over Nakagami-m Fading," IEEE Transactions on Vehicular Technology, Vol. 61, No. 5, pp. 2368 - 2374, 2012. Article (CrossRef Link)

[24] Thi My Chinh Chu; Hoc Phan; Hans-Jürgen Zepernick, “Amplify-and-forward relay assisting both primary and secondary transmissions in cognitive radio networks over Nakagami-m fading," in Proc. of IEEE 23rd International Symposium on Personal, Indoor and Mobile Radio Communications - (PIMRC), 2012. Article (CrossRef Link) 
[25] Hoc Phan; Hans-Jurgen Zepernick; Hung Tran, "Impact of interference power constraint on multi-hop cognitive amplify-and-forward relay networks over Nakagami-m fading,” IET Communications, Vol. 7, No. 9, pp. 860 - 866, 2013. Article (CrossRef Link)

[26] Mohammad M. Shurman; Mamoun F. Al-Mistarihi; Moawiah M. Alhulayil, "Performance analysis of amplify-and-forward cognitive relay networks with interference power constraints over Nakagami-m fading channels,” IET Communications, Vol. 10, No. 5, pp. 594 - 605, 2016. Article (CrossRef Link)

[27] Mohammad Shurman; Mamoun F. Al-Mistarihi; Moawiah Alhulayil, "Outage probability of dual-hop amplify-and-forward cognitive relay networks under interference power constraints over Nakagami-m fading channels," 38th International Convention on Information and Communication Technology, Electronics and Microelectronics (MIPRO), pp. 516 - 520, 2015. Article (CrossRef Link)

[28] Moawiah Alhulayil; Mamoun F. Al-Mistarihi; Mohammad Shurman, "Outage probability of amplify-and-forward underlay cognitive relay networks with selection diversity over Nakagami-m fading channels," 38th International Convention on Information and Communication Technology, Electronics and Microelectronics (MIPRO), pp. 521 - 525, 2015. Article (CrossRef Link)

[29] Rui Zhao; Yi Yuan; Lisheng Fan; Yu-Cheng He "Secrecy Performance Analysis of Cognitive Decode-and-Forward Relay Networks in Nakagami-m Fading Channels,” IEEE Transactions on Communications Year, Vol. PP, No. 99, 2016. Article (CrossRef Link)

[30] Caijun Zhong; Tharm Ratnarajah; Kai-Kit Wong, "Outage Analysis of Decode-and-Forward Cognitive Dual-Hop Systems With the Interference Constraint in Nakagami-m Fading Channels," IEEE Transactions on Vehicular Technology, Vol. 60, No. 6, pp. 2875 - 2879, 2011. Article (CrossRef Link) 


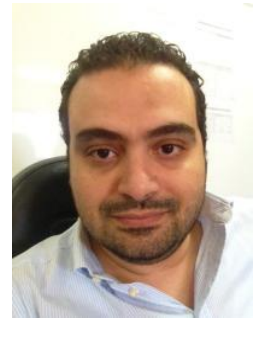

Nadhir Ben Halima received his B.Sc degree in computer engineering from the National School of Computer Sciences (ENSI), Manouba, Tunisia, the M.Sc degree in communication networks engineering from Sant'Anna School of Advanced Studies, Pisa, Italy, in 2006, and the Ph.D. degree in information and communication technology -telecommunication area -in 2009 from the University of Trento, Trento, Italy. In 2009 he was a Visiting Researcher at the Department of Electrical and Computer Engineering at North Carolina State University, Raleigh, NC, USA. Since September 2011, he is a faculty member and Vice Dean of the College of Computer Science and Engineering in Yanbu , Taibah University, Madinah, Saudi Arabia. His research interests include wireless and sensor networks, cognitive radio networks, HARQ protocoles and network security.

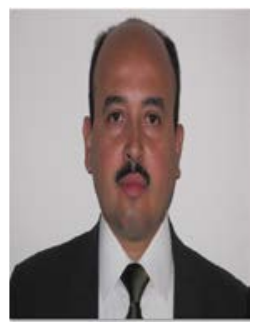

Hatem Boujemaa (M'02) was born in Tunis, Tunisia. He received the Engineer's Diploma from " Ecole Polytechnique de Tunis", in 1997, the MSC in digital communications from "Telecom Paris Tech", in 1998 and the Ph. D. degree in electronics and communications from the same university in 2001. From October 1998 to September 2001, he prepared his Ph. D. degree at France Telecom R\&D, Issy-les-Moulineaux, France. During this period, he participated in the RNRT project AUBE. From October 2001 to January 2002, he joined "Ecole Supérieure d'Electricité", Gif-sur-Yvette, France, and worked on mobile localization for RNRT project LUTECE. In February 2002, he joined SUPCOM where he is a Professor. His research activities are in the field of digital communications, DS-CDMA, OFDM and MC-CDMA systems, HARQ protocols, Cooperative Communications, Cognitive radio networks, Scheduling, Synchronization, Network planning, Information Theory, Equalization and Antenna Processing. 